\title{
Performance Analysis of Tyre Manufacturing System in the SMEs Using RAMD Approach
}

\author{
K. Velmurugan $\mathbb{D}^{1},{ }^{1}$ P. Venkumar $\mathbb{D},{ }^{1}$ and R. Sudhakarapandian $\mathbb{D}^{2}$ \\ ${ }^{1}$ Department of Mechanical Engineering, Kalasalingam Academy of Research and Education, Krishnankoil-626126, \\ Tamil Nadu, India \\ ${ }^{2}$ School of Mechanical Engineering, Vellore Institute of Technology VIT, Vellore-632014, Tamil Nadu, India
}

Correspondence should be addressed to R. Sudhakarapandian; sudhame@gmail.com

Received 31 December 2020; Revised 8 April 2021; Accepted 13 May 2021; Published 28 May 2021

Academic Editor: Abdullahi Yusuf

Copyright (c) $2021 \mathrm{~K}$. Velmurugan et al. This is an open access article distributed under the Creative Commons Attribution License, which permits unrestricted use, distribution, and reproduction in any medium, provided the original work is properly cited.

In the recent trends, production plants in the automobile industries all over the world are facing a lot of challenges to achieve better productivity and customer satisfaction due to increasing the passenger's necessity and demand for transportation. In this direction, the belt, tyre, and tube manufacturing plants act as vital roles in the day-to-day life of the automobile industries. Tyre production plant comprises five major units, namely, raw material selection, preparation, tyre components, finishing, and inspection. The main purpose of this research is to implement the new method to predict the most critical subsystems in the tyre manufacturing system of the rubber industry. As mathematically, any one maintenance parameter among reliability, availability, maintainability, and dependability (RAMD) parameters is evaluated to identify the critical subsystems and their effect on the effectiveness of the tyre production system. In this research, the effect of variation in maintenance indices, RAMD, is measured to identify the critical subsystem of the tyre production system based on the mathematical modeling Markov birth-death approach (MBDA), and the equations of the subsystems are derived by using the Chapman-Kolmogorov method. Besides, it also calculates the performance of certain maintenance parameters concerning time such as mean time between failures (MTBF), mean time to repair (MTTR), and dependability ratio for each subsystem of the tyre production system. Finally, RAMD analysis of the tyre production systems has been executed for predicting the most critical subsystem by changing the rates of failure and repair of individual subsystems with the utilization of MATLAB software. RAMD analysis reveals that the subsystem bias cutting is most critical with the minimum availability of 0.8387 , dependability 5.19 , dependability ratio 0.8701 , and maximum MTTR 38.46 hours of the subsystem. In this implementation of the proposed method, a real-time case study of the industrial repairable system of tyre manufacturing system has been taken for evaluating RAMD indices of the production plant of rubber industry cited in the southern region of Tamil Nadu, India.

\section{Introduction}

In the recent trends, in the last few decades, logistics and transportation have rapidly increased throughout the world due to the customers or passenger's necessity, and the population explosion. In that situation, the demand for the automobile industrial product (tyre, tube, and belt) manufacturing also maximized. The manufacturing process of the automobile industry consists of lots of subsystems and components. The effectiveness of the entire production system closely depends on the availability of the individual machines and their critical components [1]. The better productivity and availability of the manufacturing system can be achieved through the utilization of the proper maintenance strategy. Hence, the prediction of the critical subsystems, machines, and their components is an essential activity for a better maintenance management system in the industry. The effectiveness and performance of the production system can easily be obtained by identifying the critical subsystems, machines, and components earlier. The important maintenance parameters, RAMD, of the manufacturing system are evaluated with different selections and combinations of repair, failure rates of the individual manufacturing machines, and their components in the shop 
floor area with the utilization of the mathematical modeling and analysis. From the mathematical analysis results, significant changes in the availability of the individual subsystems, the most critical subsystem, and its components are identified. Besides, other maintenance parameters (MTBF, MTTR, dependability, and dependability ratio) are also evaluated and analyzed to identify the critical subsystems in the manufacturing system of industry. This section also presents the critical overview of the various published research articles based on the RAMD analysis of the different manufacturing systems, applications, challenges, and opportunities of mathematical modeling in the industries.

Performance evaluation of the milk production industry is described, and the reliability and availability of the milk production systems are analyzed through the application of the MINITAB software package with the different mathematical analysis techniques of RAM engineering. Also, the RAM of all individual workstations in the dairy industry is evaluated [2, 3]. RAM analysis of the reciprocating compressor in the oil and gas refinery industry has been described, and the operational performance of the compressor has been identified based on the genetic failure database with the application of the mathematical method tools and engineering techniques such as MTBF and availability of the equipment in the oil and gas industry. This research has been analyzed to develop a new model maintenance strategy to address real-time challenges in the industry's maintenance management system [4]. Reliability-centred maintenance (RCM) and redundancy allocation problem (RAP) have been discussed, and the maintenance scheduling of the repairable subsystems is analyzed through the application of three different metaheuristic algorithms such as nondominated sorting genetic algorithm (NSGA-II), multiobjective particle swarm optimization (MOPSO), and multiobjective firefly algorithm (MOFA) for implementing the novel integrated model to optimize the RCM and RAP in the industrial applications [5].

A real case study of planning the tunnel construction problems is described, and the suitable and optimal planning of the tunnel evacuation process is predicted through the simulation techniques such as Markov chain and Monte Carlo simulation techniques. With this proposed model, the optimal planning model has been implemented with reduced total cycle time of the tunnel evacuation process [6]. Implementation of the optimal Six Sigma strategy in the bag production systems through the utilization of the Define, Measure, Analyze, Improve, and Control (DMAIC) approach and RAM analysis was illustrated. This analysis estimates the nature of the downtime pattern of the bag production system concerning the RAM changes [7]. The real-time case study of the transport systems has been discussed, and the optimal travel routes are predicted based on the utilization of the three different mathematical models such as hidden Markov model, $K$-means algorithm, and Laplace smoothing techniques. The effectiveness of the route prediction is increased by organizing the optimal train model of this proposed research result [8]. Optimal maintenance and production planning for a deteriorating production system are advised using joint optimization and
Monte Carlo simulation techniques. The proposed results organize the best and optimal scheduling framework with the minimum expected cost per unit time of the production [9]. Two objectives such as addressing the uncertainty in maintenance budgeting and rehabilitation problems in the pavement have been analyzed with an application of the two stages of stochastic models in integer programming [10].

Conditional maintenance strategy simulation and sensitivity analysis model with energy consumption and carbon dioxide emissions of the production system are explained to the reduction of the total maintenance cost, environmental impact, and improvement of maintenance plan [11]. The significant issues of the real-world problems in fault detection in the industrial manufacturing process have been explained. We resolve these problems by the application of the novel method combination of the Markov Chain and generalized projection nonnegative matrix factorization (MCM GPNMF) for diagnosing the faults in the industry [12]. Reliability index analysis of the rock tunnels with the utilization of the high-dimensional model representation (HDMR) function is conducted through combining the response surface method (RSM) and first-order reliability method (FORM). The proposed model reveals the stability of the horseshoe tunnel concerning the reliability index [13]. The mathematical modeling analysis of the impact of the maintenance strategy based on the service life of the flexible pavements concerning the International Roughness Index values of the quarter heavy vehicles has been demonstrated [14]. Performance of the star repairable system with spatial dependence has been discussed, and the reliability of the systems is analyzed with the utilization of the vector Markov process and Laplace transformation methods [15].

The reliability analysis of the milk powder production system is conducted in dairy plants with the application of the Markov process and RAMD approach. The proposed results identified the critical subsystem in the milk powder production system with concerning variation in the values of major maintenance parameters [16]. Identifying the utmost critical operation in the tunnel boring systems in mixed ground conditions has been illustrated, and the RAMD of the individual tunnel boring operations in mixed ground conditions is obtained with the application of the Markov chain process [17]. Performance modeling of the refined systems in the sugar plant has been presented through the application of the Markov birth-death approach. This proposed analysis predicts the most important and sensitive subsystems in the refined system of sugar mills regarding the impact of variations on the reliability of subsystems [18]. Mathematical modeling and performance analysis of the pan crystallization system in sugar plants have been described with the application of the fuzzy reliability analysis and Markov birth-death model. The mathematical models obtain exponential distribution and differential equations of failure, and repair rates in the pan crystallization system of the sugar plant [19]. The performance and analysis of the cooking oil production plant by the application of the Markov mathematical method have been described. Besides, the most critical subsystems of the cooking oil production have been revealed concerning the shift-wise workforce in the industry [20]. 
We investigate the availability and profit of the power generation in the sewage treatment plant through the application of the MBDA with exponential distribution mathematical technique. It reveals the effect of variations in the availability changes of sewage treatment plants [21]. Performance and evaluation of channel bonding in the wireless local area network have been described. We proposed the better and optimal channel bonding with the utilization of the Markov decision model and modulation and coding scheme technology to the access point analysis of local area wireless network connection [22]. The systematic literature review of the reliability, availability, maintainability, and security/safety (RAMS) for critical infrastructure protection is explained. Identify the emerging field of research in the RAMS and critical infrastructure production technology through this critical overview research [23]. The reliability of centralized traffic control systems in the autonomous station computer system has been described. Besides, the proposed reliability analysis framework can easily predict the failure rate and fault detection probability of autonomous computer stations with the application of the Markov model and fuzzy dynamic fault tree analysis approach [24]. Variable reliability measures (RAMD) of the cloud computing technology have been explained through the application of the reliability, availability, and cost analysis approach [25]. We investigate the different types of fractional modeling problems such as falling body problems and the temperature (heating and cooling) dynamic problem in the building with the application of the Laplace transformation and Atangana-Baleanu fractional derivative in the sense of Caputo (ABC) technologies [26-28]. We investigate the transmission dynamics of pine wilt disease infections by the application of the Caputo fractional operators to analyze the biological observable behavior of the diseases. We provide more efficient and flexible information about the complexity model through the comparative analysis of the previously build integer model [29]. We identify the most critical subsystems during the conceptual designing operation in the industry through the utilization of the Markov analysis for measuring the various parameters such as failure and repair rate. This RAM analysis proposed the optimal result for predicting the sensitive subsystem in the industry concerning the variations of the parameters [30]. They have explained the evaluation of supplier selection in the industry using the hybrid fuzzy decision model for achieving the better customer satisfaction and higher productivity [31].

This research consists of an overview of the research approaches, applications, and challenges mentioned above. This research paper is based on the performance analysis and mathematical model of the tyre manufacturing system in the rubber industry to predict the most critical subsystems in the work environment using the RAMD and Markov birthdeath approach. This research technique is widely used for RAM engineering because the upcoming behaviors of a particular machine can be easily predicted by analyzing the current variables of the machine. The main motive of this research analysis is to minimize unnecessary breakdown of machines and production delay due to sudden failure of machines. Furthermore, we enhance the availability, productivity, and efficiency of the maintenance workforce through this research analysis in the rubber industry.

This research article has been organized into seven sections. The first section presents the introductory maintenance, a critical overview of the existing literature, and the motivation of this research. In Section 2, materials and methods of the proposed research have been appended. In Section 3, we deal with the assumptions, notations, and transition state diagrams of the RAMD analysis. In Section 4, we present a detailed description of the tyre manufacturing system in the rubber industry. In Section 5, numerical and graphical results of the RAMD variations are discussed through the table and graph. Section 6 presents the discussions about the RAMD findings of the tyre manufacturing system. Finally, the conclusion and future scope of this research have been illustrated in Section 7.

\section{Materials and Methods}

2.1. Markov Birth-Death Process. It is the most widely used mathematical and stochastic process to measure the maintenance parameters (RAMD) of the production systems concerning three different state conditions. This mathematical approach was introduced by the Russian mathematician Andrey Andreyevich Markov to solve sequential problems. It analyzed the present state behavior of the system to identify the future state condition of that particular system [32]. It is the best and optimal tool for the RAMD analysis because of the redundancy management of sequential arrangement problems, and it can easily predict the repairable production systems. The steady-state and transition state of the subsystems can be analyzed through the application of stochastic modeling approaches such as the Markov process and quenching process. This approach, also called Markov decision process (MDP), is further classified into the discrete and continuous state transition process (Markov process and Markov chain).

2.2. Reliability. Reliability is the most important maintenance parameter; it simply denotes " $R(t)$ " which means the probability that failure of the systems may not occur in the given specific time interval in the working environment [33]. The $R(t)$ of individual systems and subsystems concerning the rate of failure can be expressed as follows:

$$
R(t)=e^{-\lambda t}
$$

2.3. Availability. Availability of the repairable systems simply defined as the ratio of the uptime to the total lifetime of the particular production system is denoted as " $A v$," or it integrates both reliability and maintainability parameters concerning the number of failures occurring and how quickly that fault will be rectified. The $A v$ of the systems can be expressed as follows:

$$
A v=\frac{X}{X+Y} \Rightarrow \frac{\lambda}{\lambda+\mu},
$$


where $X=$ uptime or lifetime, $Y=$ downtime or repair time, and $X+Y=$ total life time.

The uptime of the systems is represented by the mean time to failure (MTTF) which can be measured through the failure analysis of the systems [33]. The mean time to repair (MTTR) can be evaluated through the maintenance analysis of the given system. The estimation of repair time is represented as follows:

$$
\begin{aligned}
A v & =\frac{M T T F}{M T T F+M T T R}, \\
M T T F & =\int R(t) \mathrm{d} t \Rightarrow \int_{0}^{\alpha} e^{-\lambda t} \mathrm{~d} t \Rightarrow \frac{1}{\lambda}, \\
M T T R & =\left(\frac{1-A v}{A v}\right) M T T F .
\end{aligned}
$$

Equation (3) expressed the availability measuring, equation (4) expressed the MTTF measuring, and equation (5) expressed the MTTR measuring of the manufacturing systems.

2.4. Maintainability. Defined as the probability function of that failed system, subsystems and their components are restored into the original or operative condition in the given specific time interval (downtime) that is denoted as " $M(t)$ " [34]. The $M(t)$ of the industrial repairable systems can be expressed as follows:

$$
M(t)=1-e^{-\mu t}
$$

2.5. Dependability. Dependability is another most important maintenance parameter measure; it is simply defined as the system's ability to deliver the service with a specific time interval concerning the assumption of the rate of failure and repair of the production system [35]. The dependability and its ratio " $D_{\text {Min }}$ " of the repairable industrial system can be expressed as follows:

$$
\begin{aligned}
d & =\frac{M T T F}{M T T R} \\
\operatorname{Dmin}_{i} & =\left[1-\left(\frac{1}{d-1}\right)\left(e^{-\ln d /(d-1)}-e^{-d \ln d /(d-1)}\right)\right] .
\end{aligned}
$$

\section{Notations and Assumptions}

The notations and assumptions used for RAMD analysis of the tyre manufacturing model are provided. The tyre manufacturing system of the rubber industry is described briefly, and maintenance problems of this system are discussed. Achieving better availability of production machinery and its equipment at this shop floor is one of the major problems of the rubber industry. The transition state diagram of the tyre manufacturing machines is illustrated in Figure 1 .

3.1. Notations. The various notations used for this RAMD analysis research in the tyre manufacturing system of the rubber industry are given as follows:

SBM, SBB, SCL, SBC, SEX, SBD, and SCU working state of the machines

SBM, SBB, SCL, SBC, SEX, SBD, and SCU under maintenance state of the machines

$* \mathrm{SBM}, * \mathrm{SBB}, * \mathrm{SCL}, * \mathrm{SBC}, * \mathrm{SEX}, * \mathrm{SBD}$, and $* \mathrm{SCU}$ repair state of the machines

BM: Banbury mixing subsystem

BB: bead and belt cord manufacturing subsystem

$\mathrm{CL}$ : calendaring subsystem

BC: bias cutting subsystem

EX: extruding subsystem

BD: building subsystem

CU: curing subsystem

$\lambda_{i}$ : failure rate of the subsystems $(i=\mathrm{BM}, \mathrm{BB}, \mathrm{CL}, \mathrm{BC}$, $\mathrm{EX}, \mathrm{BD}$, and $\mathrm{CU}$ )

$\mu_{i}$ : repair rate of the subsystems

$\phi_{i}$ : transition rate of the subsystems

$\eta_{i}$ : PM rate of the subsystems

$b$ : constant ( 0 for ideal and 1 for faulty)

$P_{0}(t)$ : the probability function of subsystems is in the original state

$P_{1}(t)$ : the probability functions of the respective subsystems are in the under maintenance state

$P_{2}(t)$ : the probability functions of the respective subsystems are in the repair state

3.2. Assumptions. Here, the RAMD analysis of the tyre manufacturing system maintenance model in this research article confirms the following assumptions:

(i) Initially, every subsystem is in the original state or $\operatorname{good}(\mathrm{BM}, \mathrm{BB}, \mathrm{CL}, \mathrm{BC}, \mathrm{EX}, \mathrm{BD}$, and $\mathrm{CU})$

(ii) The rate of repair and failure of every subsystem is constant and statistically independent $\left(\lambda_{i}\right.$, $\mu_{i}=$ constant $)(i=\mathrm{BM}, \mathrm{BB}, \mathrm{CL}, \mathrm{BC}, \mathrm{EX}, \mathrm{BD}$, and $\mathrm{CU})$

(iii) Every repaired system is considered as good as new

(iv) The single maintenance team to handle the PM of the subsystem

(v) Every subsystem has three state conditions such as original, under maintenance, and repair (e.g., S, S, and $* \mathrm{~S}$ )

(vi) The simultaneous failures of the subsystems are not considered

(vii) The rate of PM and transition of the critical systems are taken as constant ( $\eta i, \phi_{i}=$ constant) 


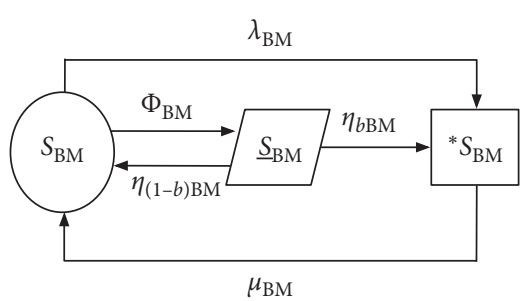

(a)

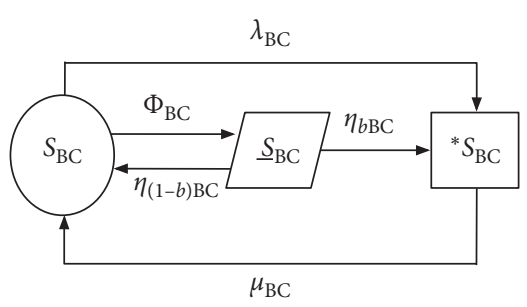

(d)

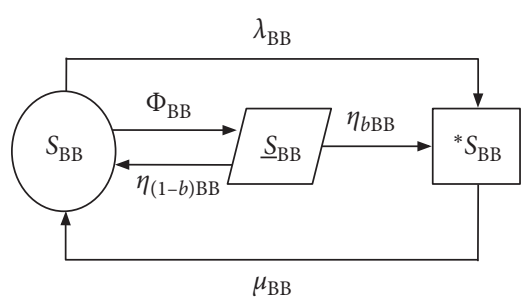

(b)

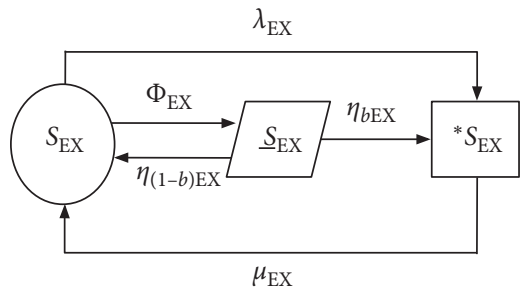

(e)

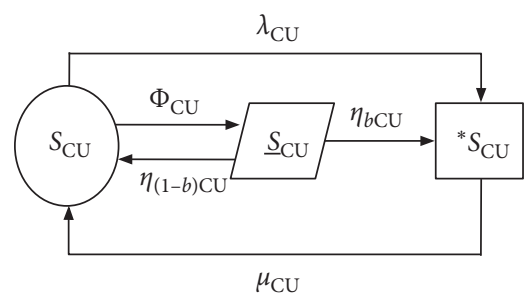

(g)

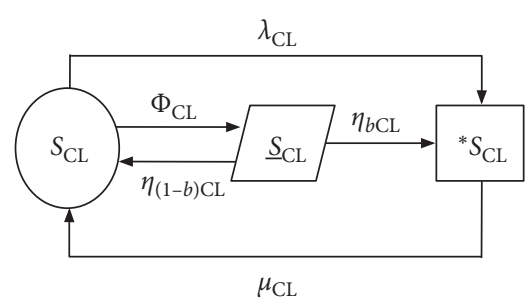

(c)

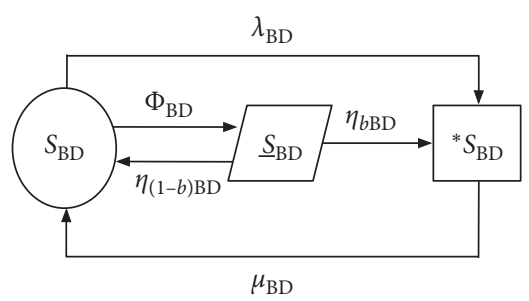

(f)

FIgURE 1: Transition state diagram of tyre production systems.

\section{Description of Tyre Manufacturing System}

In this research, all the maintenance parameters measured are derived on the subsystem wise. The rate of failure and repair of all the subsystems are considered exponentially distributed. In this section, a detailed description of the tyre manufacturing plant has been presented. The tyre manufacturing plant has five major systems such as material selection, initial preparation, tyre component preparation, finishing, and inspections. The graphical representation of the tyre manufacturing system is illustrated in Figure 2. These systems majorly considered several subsystems such as Banbury mixing, bead and belt cord manufacturing, calendaring, bias cutting, extruding, building, and curing. All the subsystems are arranged in the series operation architecture, and these subsystems have internal redundancy. A detailed explanation of the tyre production systems is given as follows.

4.1. System A (Raw Materials). The tyre production system consists of these major materials such as rubber, textiles, carbon black, sulfur, and other chemical additives. Rubber is the major raw material (natural and synthetic rubber), and it has been collected from various places such as Kerala. The other ingredient is carbon black which is in a fine powder form. It has been added to the raw rubber for the softening process in the tyre production system. Then, sulfur and some chemical additives are added into the raw rubber to achieve the required tyre characteristics such as friction and ultraviolet radiation.

4.2. System B (Preparation). The tyre manufacturing process preparation is the initial operation, and it has some series operating subsystems such as Banbury mixing and bead and belt cord manufacturing. A detailed description of the subsystems is given below.

4.2.1. Banbury Mixing (Subsystem BM). It is the initial process of the tyre manufacturing system, the mixing of raw materials (natural and synthetic rubber, carbon black, sulfur, and other chemical additives), and forming the rubber compound through the application of the computer control system. That controls the composition of rubber, and chemical mixing automatically depends upon the required tyre parts.

4.2.2. Bead and Belt Cord Manufacturing (Subsystem BB). In this section, the mixed rubber compounds are further heated for softening the mix and evenly distributing the chemical additives. Once it is completed, that mixed compound feeds into the two sets of rollers and rotates the different directions of the powerful rolling mill that presses and squeezes the mixed rubber compound and makes the thick sheet. Those sheets are utilized to produce the specific part (bead and belt cord) of the tyre components covered 


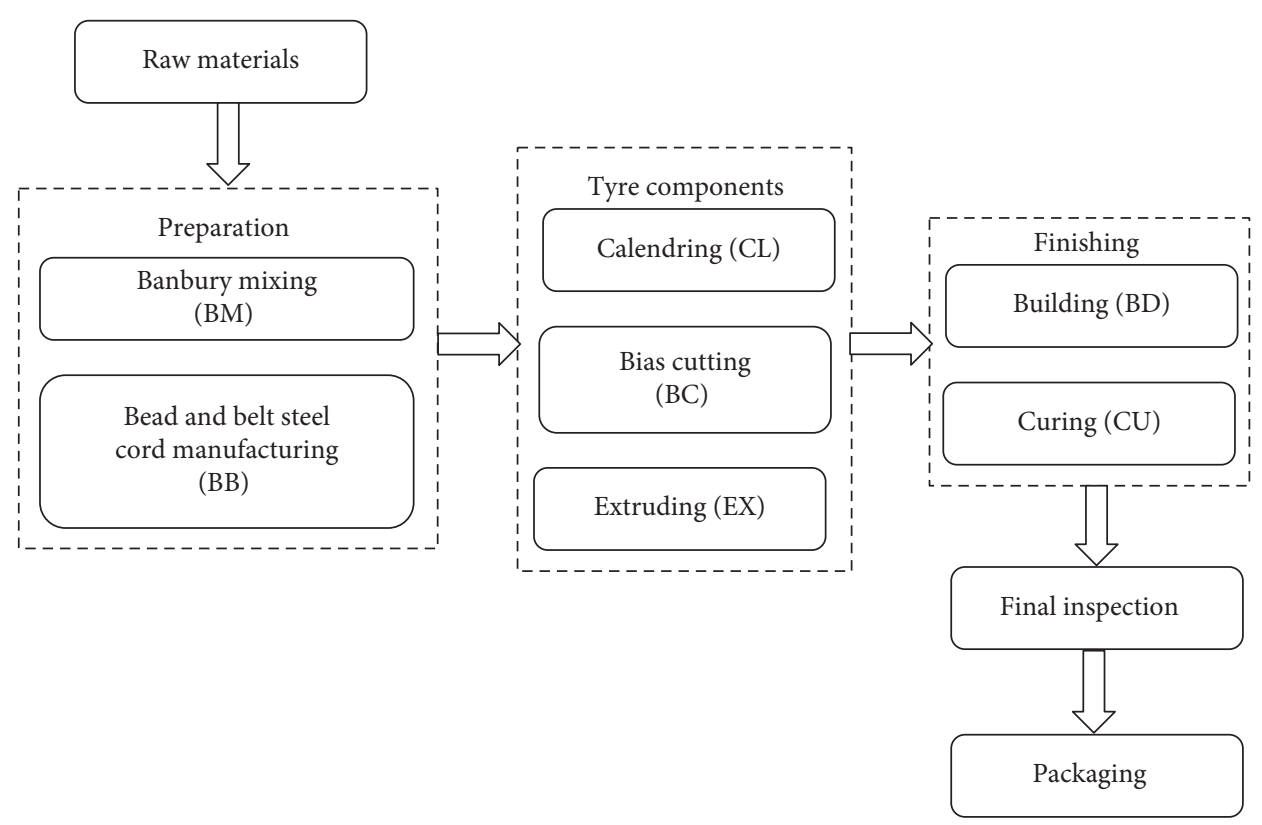

FIgURE 2: Manufacturing process of a tyre production system.

with a combination of textile and fabric. This final rubberized fabric was used to build the layer of the tyre called a ply.

4.3. System C(Tyre Components). This section consists of the three different subsystems that the manufacturing process applied to produce the tyre components. The detailed description of the tyre component production subsystems is explained below.

4.3.1. Calendaring (Subsystem CL). After completing the mixed rubber compounds that will feed into the calendering operation for reducing the thickness of the rubber-mixed compound, we released the porous gases and gravidities in the rubber-mixed compounds through the application of the powerful rolling operation. This calendaring subsystem has some major activities such as (1) to create the mixed rubber compound as a uniform thick sheet with specific dimensions and (2) to prepare the initial coats of rubber on the textile fabric, to insert the steel or textile fabric in the rubber compound through friction.

4.3.2. Extruding (Subsystem EX). In the extruding operations, the mixed rubber compound feeds into the extruder machine and then forced out through the die with the required shape of an orifice to produce the required size and shape of the tyre components. The large, flat sheet of the tyre treads is developed by the extruding process.

4.3.3. Bias Cutting (Subsystem BC). After the calendaring and extruding operations are completed, flat rubber sheets move into the conveyor to the bias cutting operation. In that situation, we cut the required shape and size of the flat rubber sheets with the application of the grating blade.
4.4. System D (Finishing). This section consists of the two subsystems of the final operation in the tyre manufacturing process. The detailed demonstration of the subsystems is given as follows.

4.4.1. Building (Subsystem BD). This system is also called the tyre assembly process after the bias cutting process that stripped textile fabric rubber compound sheets, and other tyre components (bread, plies, sidewall, and treads) are assembled on the drum of the building machine to form the "green tyre" through this operation.

4.4.2. Curing (Subsystem CU). Finally, that green tyre goes to the vulcanizing process that is placed on the large mold of the curing machine that is called the bladder. That bladder filled with the high pressure and temperature steams during this operation green tyre is vulcanized up to $280^{\circ}$ within the specified time limit. After completing the curing operation, the finished tyre will go to the cooling process and then quality checking activity.

4.5. System E (Inspection). After the vulcanizing process is completed, the finished tyre is moved into the quality and inspection process. In that situation, each tyre is thoroughly inspected with the application of various techniques such as visual inspection, destructive, and nondestructive inspection techniques, and based on these techniques, the flaws are identified such as bubbles and voids in the tyres. Once they completed the inspection and satisfy the required quality, the final product is moved into the packaging section and then stored in the warehouse for customer distribution. 


\section{Numerical and Graphical Result}

The real-time case study of the RAMD analysis in the tyre manufacturing system of the rubber industry has been evaluated by applying the last five-year maintenance record data of the individual subsystems in the tyre manufacturing system [36, 37]. The input numerical values of RAMD analysis for applying the mathematical modeling are illustrated in Table 1.

5.1. RAMD Indices of the Tyre Manufacturing Subsystems. This research analyzed the performance of the tyre production systems in the rubber industry. The description of the tyre production system is presented in the previous section. That production system has seven numbers of subsystems to achieve tyre production. All the subsystems are arranged in the series configuration production process in the manufacturing plant. Hence, the maintenance parameters (RAMD) of all the subsystems are measured through the utilization of the Markov birth-death process and the mathematical equations are derived by using the differential-difference equations [36, 37]. The basis of the Markov birth-death approach sample transition state diagram and the mathematical modeling of the reference subsystems is graphically represented in Figure 3. The recurrence relationship of subsystems with derived mathematical equations is as follows:

$$
\begin{aligned}
& P_{0}^{\prime}(t)=\eta_{i(1-b)} P_{0}(t)+\mu_{i} P_{2(t)}-\varphi_{i} P_{0(t)}-\lambda_{i} P_{0(t)}, \\
& P_{1}^{\prime}(t)=\varphi_{i} P_{0}(t)-\eta_{i(1-b)} P_{1(t)}-\eta_{i b} P_{1(t)}, \\
& P_{2}^{\prime}(t)=\eta_{i b} P_{1(t)}+\lambda_{i} P_{0}(t)-\mu_{i} P_{2(t)} .
\end{aligned}
$$

In initial state conditions, apply time $t=0$ in equations (1) to (3). We get

$$
\begin{array}{r}
\eta_{i(1-b)} P_{0}+\mu_{i} P_{2}-\varphi i P_{0}-\lambda_{i} P_{0}=0, \\
\varphi i P_{0}-\eta_{i(1-b)} P_{1}-\eta_{i b} P_{1}=0, \\
\eta_{i b} P_{1}+\lambda_{i} P_{0}-\mu_{i} P_{2}=0 .
\end{array}
$$

Now, applying the normalizing state conditions where $j=0,1$, and 2 ,

$$
\sum_{j}^{n} P j=1 \Rightarrow P_{0}+P_{1}+P_{2}=1 .
$$

Derive equations (1) to (3), and substitute the values of $P_{0}, P_{1}$, and $P_{2}$ in equation (8). The availability of the tyre manufacturing subsystems is as follows:

$$
A V_{i}=\left[1+\left(\frac{\varphi_{i}}{\eta_{i(1-b)}+\eta_{i b}}\right)+\left(\frac{\varphi_{i} \times \eta_{i b}+\lambda_{i}}{\mu_{i}\left(\eta_{i(1-b)}+\eta_{i b}\right)}\right)\right]^{-1},
$$

where $i=\mathrm{BM}, \mathrm{BB}, \mathrm{CL}, \mathrm{BC}, \mathrm{EX}, \mathrm{BD}$, and CU, $t=$ time, and if $b=1$ for faulty state condition and if $b=0$ for ideal state condition, availability of the tyre manufacturing subsystem in fault state condition is $S_{\mathrm{BM}}=0.8827$. Availability of the tyre manufacturing subsystem in ideal state condition is $S_{\mathrm{BM}}=0.9206$. Reliability of the tyre manufacturing subsystems is derived as follows:

$$
\begin{aligned}
R_{i}(t) & =e^{-\lambda_{i} t}, \\
R B M(t) & =e^{-0.008 t} .
\end{aligned}
$$

Maintainability of the tyre manufacturing subsystems is derived as follows:

$$
\begin{aligned}
M_{i}(t) & =1-e^{-\mu_{i} t}, \\
M_{\mathrm{BM}}(t) & =1-e^{-0.150 t} .
\end{aligned}
$$

Other maintenance performance parameter measures of the tyre manufacturing subsystems are as follows:

$$
\begin{aligned}
\operatorname{MTBF}_{i} & =\frac{1}{\lambda_{i}} ; \\
\operatorname{MTTR}_{i} & =\frac{\operatorname{MTBF}_{i}\left(1-A v_{i}\right)}{A v_{i}}, \\
d_{i} & =\frac{M T B F_{i}}{M T T R_{i}} ; \\
\operatorname{Dmin}_{i} & =\left[1-\left(\frac{1}{d-1}\right)\left(e^{-\ln d /(d-1)}-e^{-d \ln d /(d-1)}\right)\right],
\end{aligned}
$$

where $M T B F_{\mathrm{BM}}=125.00 ; M T T R_{\mathrm{BM}}=16.16 ; d_{\mathrm{BM}}=7.52$; and $D_{\min (\mathrm{BM})}=0.9025$.

Similarly, we measured the all other individual subsystem maintenance parameters through the application of equations (8) to (18) and the transition state diagram of the individual subsystems are illustrated in Figure 1 for analyzing the total performance of the tyre manufacturing system in the rubber industry.

5.2. Reliability of the System. Since the seven subsystems are arranged in the series configuration of the tyre manufacturing system and if failed, any one of the subsystems causes the entire production system to fail. The reliability of the tyre production system is equal to the product of each subsystem's reliability. Hence, the overall reliability of the tyre production systems is achieved by using the following equation:

$$
\begin{aligned}
R_{\text {Sys }}(t)= & R_{B M}(t) \times R_{B B}(t) \times R_{C L}(t) \times R_{B C}(t) \times R_{E X}(t) \\
& \times R_{B D}(t) \times R_{C U}(t), \\
R_{\text {Sys }}(t)= & e^{-0.008 t} \times e^{-0.007 t} \times e^{-0.004 t} \times e^{-0.005 t} \times e^{-0.007 t} \\
& \times e^{-0.003 t} \times e^{-0.002 t}, \\
R_{\text {Sys }}(t)= & e^{-0.036 t} .
\end{aligned}
$$

The effect of changes of all the subsystems and total system reliability concerning the time between 30 and 75 days are analyzed, and results are illustrated in Table 2 . The 
TABLE 1: Input numerical values of the RAMD analysis.

\begin{tabular}{|c|c|c|c|c|}
\hline Subsystem & Failure rate & Repair rate & Transition rate & PM rate \\
\hline Banbury mixing & 0.008 & 0.150 & 0.007 & 0.70 \\
\hline Bead and belt cord making & 0.007 & 0138 & 0.005 & 0.60 \\
\hline Calendaring & 0.004 & 0.290 & 0.006 & 0.40 \\
\hline Bias cutting & 0.005 & 0.158 & 0.003 & 0.20 \\
\hline Extruding & 0.007 & 0.144 & 0.008 & 0.50 \\
\hline Building & 0.003 & 0.244 & 0.004 & 0.30 \\
\hline Curing & 0.002 & 0.310 & 0.002 & 0.20 \\
\hline
\end{tabular}

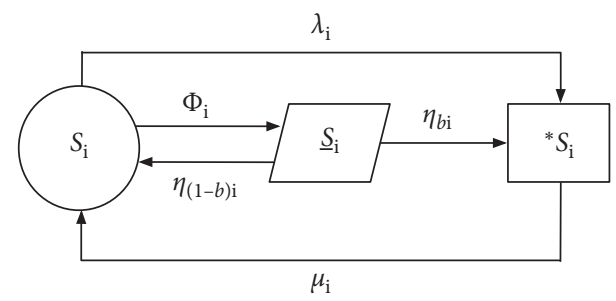

Figure 3: Sample transition state diagram of the subsystem.

reliability variation of the tyre production system pictorial representation is presented in Figure 4. In the graphical representation, horizontal axis denotes the time in days and the vertical axis refers to the reliability of the individual subsystem and entire tyre production system. The reliability of the individual subsystems in the tyre production system has gradually decreased concerning the working time.

5.3. Availability of the System. The availability of the individual subsystems will affect the entire production system because of the series configuration of the manufacturing process in the industry. The availability of the tyre production system equals the product of the individual subsystem's availability. Hence, the overall availability of the tyre production system is demonstrated in the following equation:

The effect of changes of all the subsystems and total system availability concerning the specific rate of failure and repair of the subsystems are analyzed, and results are illustrated in Table 3 . The availability variation of the tyre production system graphical representation is presented in Figure 5. In the pictorial representation, the horizontal line denotes the subsystem of the tyre production and the vertical line refers to the availability of the particular subsystem. The spline curve denotes the two different states (faulty and ideal) of availability variations of the individual subsystems in the tyre production system.

$$
\begin{aligned}
A V_{\text {sys }}= & A V_{\mathrm{BM}} \times A V_{\mathrm{BB}} \times A V_{\mathrm{CL}} \times A V_{\mathrm{BC}} \times A V_{\mathrm{EX}} \\
& \times A V_{\mathrm{BD}} \times A V_{\mathrm{CU}}, \\
A V_{\text {sys }} \text { at Faulty }= & 0.8827 \times 0.8859 \times 0.9344 \times 0.8387 \\
& \times 0.8555 \times 0.9339 \times 0.9535=0.4668, \\
A V_{\text {sys }} \text { at Faulty }= & 0.9206 \times 0.9153 \times 0.9528 \times 0.8523 \\
& \times 0.8982 \times 0.9485 \times 0.9495=0.5535 .
\end{aligned}
$$

5.4. Maintainability of the System. The overall maintainability of the tyre production system can be obtained by utilizing the following equation:

$$
\begin{aligned}
M_{\text {sys }}(t)= & M_{\mathrm{BM}}(t) \times M_{\mathrm{BB}}(t) \times M_{\mathrm{CL}}(t) \times M_{\mathrm{BC}}(t) \\
& \times M_{\mathrm{EX}}(t) \times M_{\mathrm{BD}}(t) \times M_{\mathrm{CU}}(t), \\
M_{\text {sys }}(t)= & \left(1-e^{-0.150 t}\right) \times\left(1-e^{-0.138 t}\right) \times\left(1-e^{-0.290 t}\right) \\
& \times\left(1-e^{-0.158 t}\right) \times\left(1-e^{-0.144 t}\right) \times\left(1-e^{-0.244 t}\right) \\
& \times\left(1-e^{-0.310 t}\right)=\left(1-e^{-1.43 t}\right) .
\end{aligned}
$$

The effect of changes of all the subsystems and total system maintainability concerning the time between 30 and 75 days are analyzed, and results are illustrated in Table 4. 
TABLE 2: Effect of changes in the reliability of tyre production system.

\begin{tabular}{|c|c|c|c|c|c|c|c|c|}
\hline$T$ (days) & $R_{\mathrm{BM}}$ & $R_{\mathrm{BB}}$ & $R_{\mathrm{CL}}$ & $R_{\mathrm{BC}}$ & $R_{\mathrm{EX}}$ & $R_{\mathrm{BD}}$ & $R_{\mathrm{CU}}$ & $R_{\text {Sys }}$ \\
\hline 30 & 0.7866 & 0.8106 & 0.8869 & 0.8607 & 0.8106 & 0.9139 & 0.9418 & 0.3396 \\
\hline 35 & 0.7558 & 0.7827 & 0.8694 & 0.8395 & 0.7827 & 0.9003 & 0.9324 & 0.2837 \\
\hline 40 & 0.7261 & 0.7558 & 0.8521 & 0.8187 & 0.7558 & 0.8869 & 0.9231 & 0.2369 \\
\hline 45 & 0.6977 & 0.7298 & 0.8353 & 0.7985 & 0.7298 & 0.8737 & 0.9139 & 0.1979 \\
\hline 50 & 0.6703 & 0.7047 & 0.8187 & 0.7788 & 0.7047 & 0.8607 & 0.9048 & 0.1653 \\
\hline 55 & 0.6440 & 0.6805 & 0.8025 & 0.7596 & 0.6805 & 0.8479 & 0.8958 & 0.1381 \\
\hline 60 & 0.6188 & 0.6570 & 0.7866 & 0.7408 & 0.6570 & 0.8353 & 0.8869 & 0.1153 \\
\hline 65 & 0.5945 & 0.6344 & 0.7711 & 0.7225 & 0.6344 & 0.8228 & 0.8781 & 0.0963 \\
\hline 70 & 0.5712 & 0.6126 & 0.7558 & 0.7047 & 0.6126 & 0.8106 & 0.8694 & 0.0805 \\
\hline 75 & 0.5488 & 0.5916 & 0.7408 & 0.6873 & 0.5916 & 0.7985 & 0.8607 & 0.0672 \\
\hline
\end{tabular}

The maintainability variation of the tyre production system graphical representation is presented in Figure 6. The horizontal axis denotes the working time in days, and the vertical axis represents the maintainability of the entire tyre production system in the industry.

5.5. Dependability of the System. The overall dependability of the series configured tyre production system can be obtained by utilizing the following equation:

$$
\begin{aligned}
D_{\mathrm{Sys}(\min )}(t)= & D_{\mathrm{BM}(\min )}(t) \times D_{\mathrm{BB}(\min )}(t) \times D_{\mathrm{CL}(\min )}(t) \\
& \times D_{\mathrm{BC}(\min )}(t) \times D_{\mathrm{EX}(\min )}(t) \times D_{\mathrm{BD}(\min )}(t) \\
& \times D_{\mathrm{CU}(\min )}(t), \\
D_{\mathrm{Sys}(\min )}= & 0.9025 \times 0.9049 \times 0.9429 \times 0.8701 \\
& \times 0.8823 \times 0.9422 \times 0.9582=0.53370 .
\end{aligned}
$$

5.6. Mean Time to Repair. The MTTR of repairable tyre production subsystems concerning the specific availability changes in the subsystems of the tyre production systems is derived through the application of equations (5) or (9). The graphical representation of the MTTR variation is shown in Figure 7. In the pictorial representation, the horizontal axis denotes the individual subsystem of the tyre production and the vertical axis refers MTTR of that particular subsystem. The individual subsystem's MTTR variation can be obtained by applying the following equation:

$$
\begin{aligned}
& \operatorname{MTTR}_{\mathrm{BM}}=\frac{M T B F_{\mathrm{BM}}\left(1-A v_{\mathrm{BM}}\right)}{A v_{\mathrm{BM}}}, \\
& \operatorname{MTTR}_{\mathrm{BM}}=\frac{125(1-0.8827)}{0.8827} \Rightarrow 16.16 \mathrm{Hr} .
\end{aligned}
$$

The summary of all the subsystem maintenance parameters of the tyre production system in the rubber industry is appended in Table 5.

\section{Discussion}

The RAMD analysis of the numerous subsystems and tyre production systems has been carried out for a real-time case study of the small- and medium-scale rubber industry by assigning the numerical values of the maintenance parameters of the tyre production system as shown in Table 1 . The $R(t), A v, M(t), D_{\text {Min }}$, and MTTR of the individual subsystems of the tyre production plant have been revealed in Tables 2-4, and 6. The overall RAMD indices of the tyre production systems concerning the numerical values and the mathematical modeling results have been illustrated in Table 5. The maintenance parameters availability, dependability, and dependability ratio values of the subsystem BM (Banbury mixing) are lower than those of the other subsystems in the overall tyre production system while the MTTR of the particular subsystem is higher than that of all other subsystems. Hence, the more attention is needed to the subsystem BM compared to all other subsystems for achieving the better performance and availability of the tyre production system in the rubber industry. The effect of rate of failure and repair of the subsystem BM on the availability of that particular subsystem is revealed by varying the repair rate of the subsystem $\mathrm{BC} \mu_{\mathrm{BC}}=0.128$ to 0.188 , a failure rate of the subsystem $\mathrm{BC} \lambda_{\mathrm{BC}}=0.002$ to 0.008 , and other maintenance parameters such as transition rate and preventive maintenance rate of the subsystem $\mathrm{BC}$ are taken as a constant value $\eta_{\mathrm{BC}}=0.003$ and $\phi_{\mathrm{BC}}=0.2$. The availability of the subsystem $\mathrm{BC}$ has been calculated through the application of these values, and results are illustrated in Table 7.

That result shows the availability of the subsystem BC approximately decreases from 3.03 to $2.21 \%$ with an increased repair rate of the particular subsystem. However, the availability of the subsystem will increase from 0.59 to $1.36 \%$ with an increased failure rate of the subsystem, and the MTTR will decrease by $4.87 \%$ approximately. The graphical representation of the availability changes in the subsystem $\mathrm{BC}$ concerning the rate of failure and repair is shown in Figure 8. The $A v$ variation of the subsystem $\mathrm{BC}$ is entirely different from all other subsystems due to the sudden up and down values of (up to normal) $A v$ changes. Other 


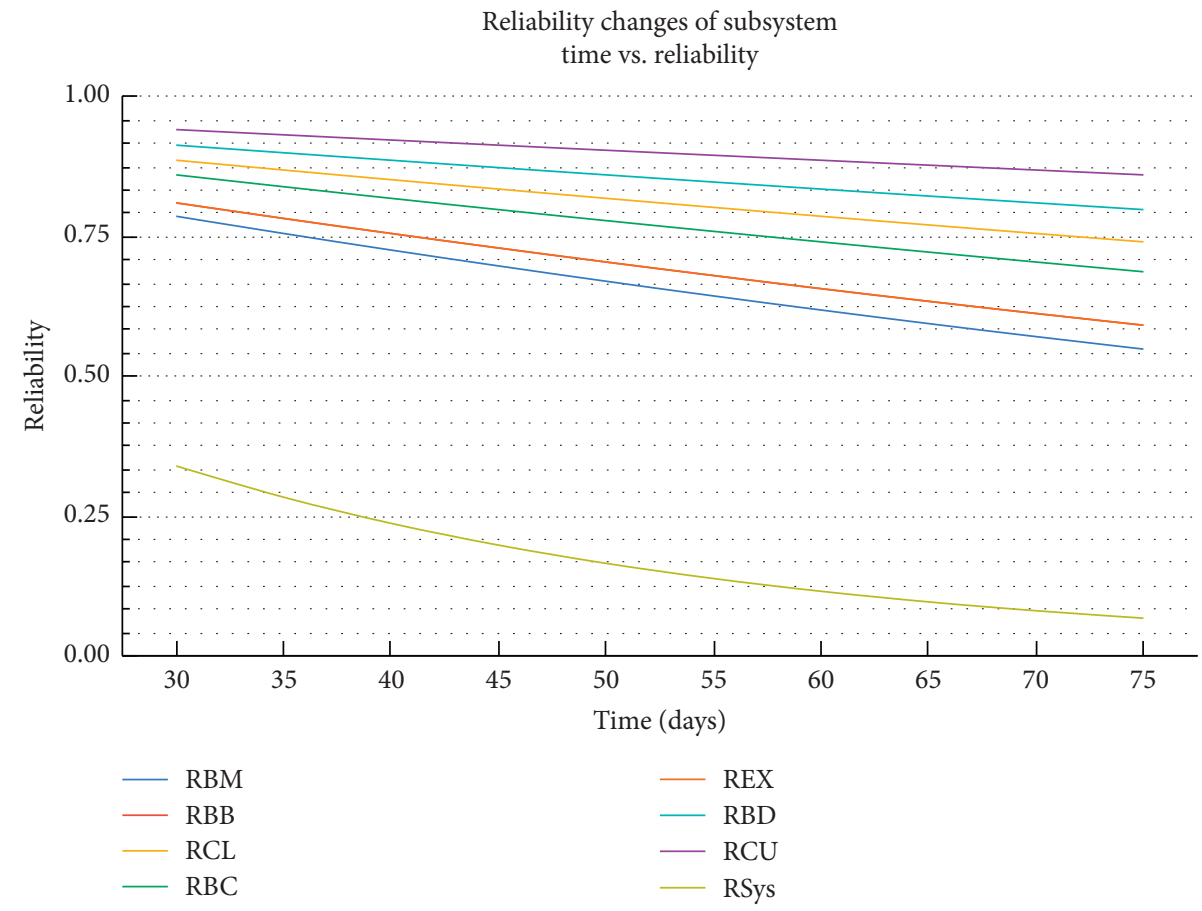

FIGURE 4: Reliability changes of the tyre production system.

TABLE 3: Effect of changes in the availability of tyre production system.

\begin{tabular}{lcc}
\hline Subsystem & Availability at faulty state & Availability at ideal state \\
\hline Banbury mixing & 0.8827 & 0.9206 \\
Bead and belt cord making & 0.8859 & 0.9153 \\
Calendaring & 0.9344 & 0.9528 \\
Bias cutting & 0.8387 & 0.8523 \\
Extruding & 0.8555 & 0.8982 \\
Building & 0.9339 & 0.9485 \\
Curing & 0.9535 & 0.9594 \\
\hline
\end{tabular}

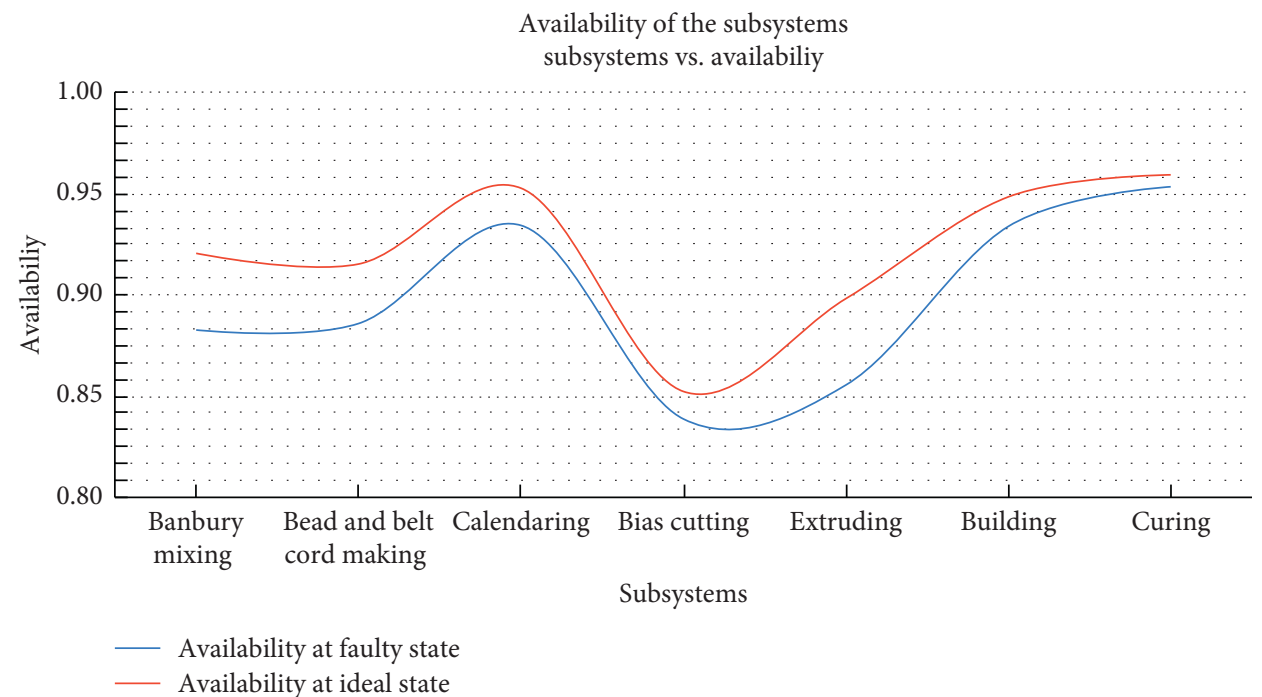

Figure 5: Availability variation of the tyre production system. 
TABLE 4: Effect of changes in the maintainability of a tyre production system.

\begin{tabular}{|c|c|c|c|c|c|c|c|c|}
\hline$T$ (days) & $M_{\mathrm{BM}}$ & $M_{\mathrm{BB}}$ & $M_{\mathrm{CL}}$ & $M_{\mathrm{BC}}$ & $M_{\mathrm{EX}}$ & $M_{\mathrm{BD}}$ & $M_{\mathrm{CU}}$ & $M_{\text {Sys }}$ \\
\hline 30 & 0.9889 & 0.9841 & 0.9998 & 0.9913 & 0.9867 & 0.9993 & 0.9999 & 1.0000 \\
\hline 35 & 0.9948 & 0.9920 & 1.0000 & 0.9960 & 0.9935 & 0.9998 & 1.0000 & 1.0000 \\
\hline 40 & 0.9975 & 0.9960 & 1.0000 & 0.9982 & 0.9968 & 0.9999 & 1.0000 & 1.0000 \\
\hline 45 & 0.9988 & 0.9980 & 1.0000 & 0.9992 & 0.9985 & 1.0000 & 1.0000 & 1.0000 \\
\hline 50 & 0.9994 & 0.9990 & 1.0000 & 0.9996 & 0.9993 & 1.0000 & 1.0000 & 1.0000 \\
\hline 55 & 0.9997 & 0.9995 & 1.0000 & 0.9998 & 0.9996 & 1.0000 & 1.0000 & 1.0000 \\
\hline 60 & 0.9999 & 0.9997 & 1.0000 & 0.9999 & 0.9998 & 1.0000 & 1.0000 & 1.0000 \\
\hline 65 & 0.9999 & 0.9999 & 1.0000 & 1.0000 & 0.9999 & 1.0000 & 1.0000 & 1.0000 \\
\hline 70 & 1.0000 & 0.9999 & 1.0000 & 1.0000 & 1.0000 & 1.0000 & 1.0000 & 1.0000 \\
\hline 75 & 1.0000 & 1.0000 & 1.0000 & 1.0000 & 1.0000 & 1.0000 & 1.0000 & 1.0000 \\
\hline
\end{tabular}

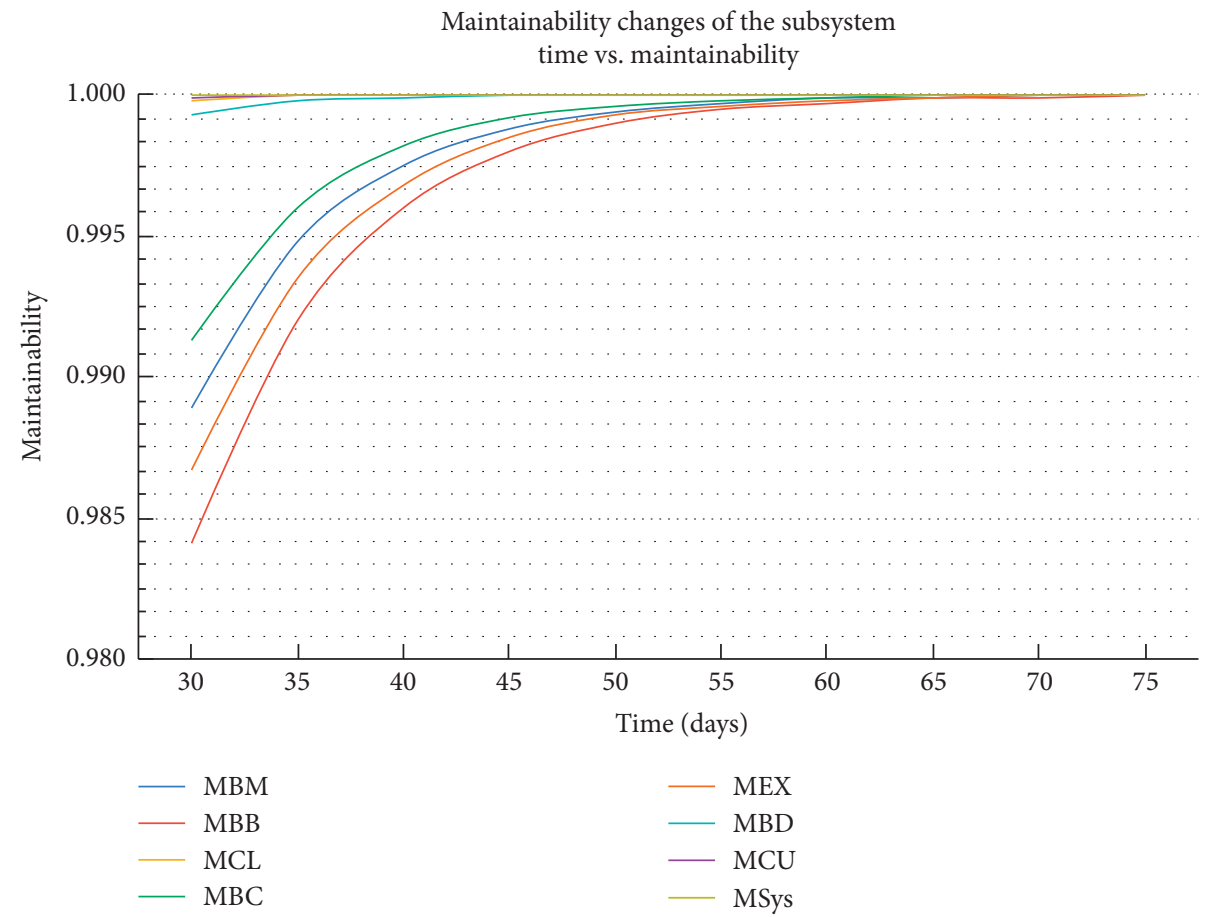

FIgURE 6: Maintainability changes of a tyre production system.

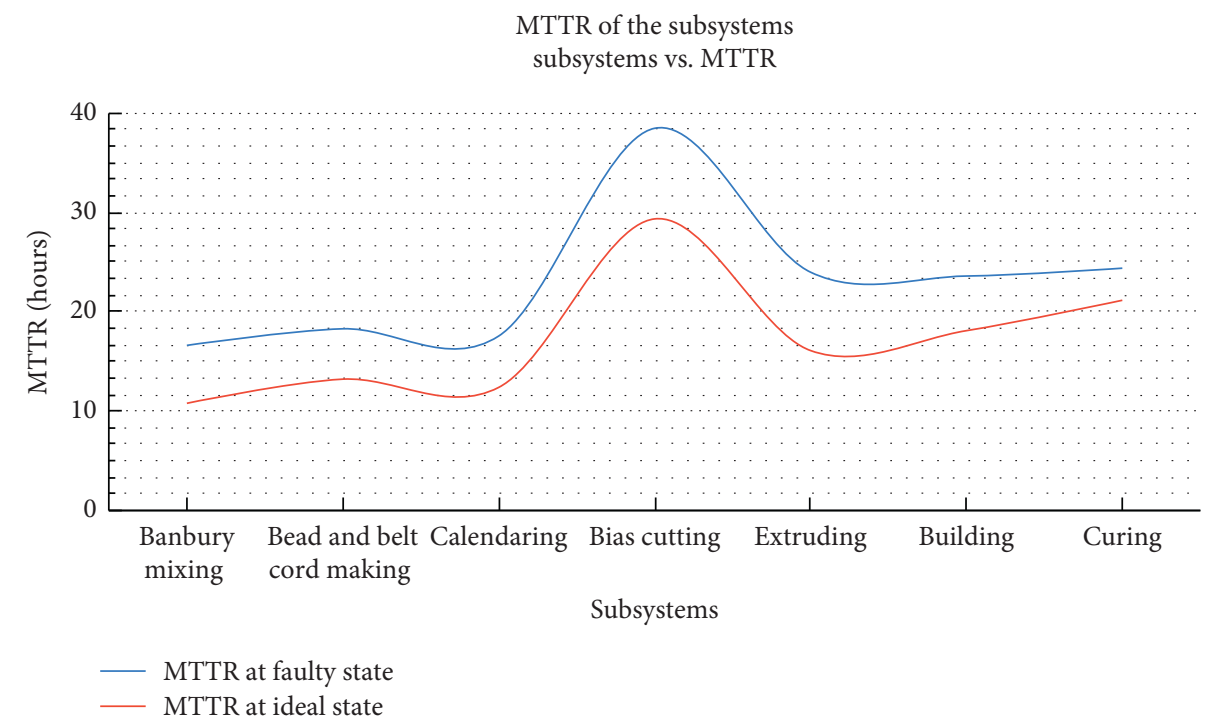

FIGURE 7: MTTR changes in the tyre production system. 
TABLE 5: RAMD indices for the tyre production system.

\begin{tabular}{|c|c|c|c|c|c|c|c|}
\hline RAMD indices & $S_{\mathrm{BM}}$ & $S_{\mathrm{BB}}$ & $S_{\mathrm{CL}}$ & $S_{\mathrm{BC}}$ & $S_{\mathrm{EX}}$ & $S_{\mathrm{BD}}$ & $S_{\mathrm{CU}}$ \\
\hline Reliability & $e^{-0.008 t}$ & $e^{-0.007 t}$ & $e^{-0.004 t}$ & $e^{-0.005 t}$ & $e^{-0.007 t}$ & $e^{-0.003 t}$ & $e^{-0.002 t}$ \\
\hline Maintainability & $1-e^{-0.150 t}$ & $1-e^{-0.138 t}$ & $1-e^{-0.290 t}$ & $1-e^{-0.158 t}$ & $1-e^{-0.144 t}$ & $1-e^{-0.244 t}$ & $1-e^{-0.310 t}$ \\
\hline Availability (faulty) & 0.8827 & 0.8859 & 0.9344 & 0.8387 & 0.8555 & 0.9339 & 0.9535 \\
\hline Availability (ideal) & 0.9206 & 0.9153 & 0.9528 & 0.8523 & 0.8982 & 0.9485 & 0.9594 \\
\hline Dependability & 7.52 & 7.76 & 14.24 & 5.19 & 5.92 & 14.12 & 20.5 \\
\hline MTTR (faulty) & $16.61 \mathrm{~h}$ & $18.28 \mathrm{~h}$ & $17.55 \mathrm{~h}$ & $38.46 \mathrm{~h}$ & $23.98 \mathrm{~h}$ & $23.59 \mathrm{~h}$ & $24.38 \mathrm{~h}$ \\
\hline MTTR (ideal) & $10.78 \mathrm{~h}$ & $13.21 \mathrm{~h}$ & $12.38 \mathrm{~h}$ & $29.34 \mathrm{~h}$ & $16.09 \mathrm{~h}$ & $18.09 \mathrm{~h}$ & $21.15 \mathrm{~h}$ \\
\hline MTBF & $125.0 \mathrm{~h}$ & $142.8 \mathrm{~h}$ & $250.0 \mathrm{~h}$ & $200.0 \mathrm{~h}$ & $142.0 \mathrm{~h}$ & $333.3 \mathrm{~h}$ & $500.0 \mathrm{~h}$ \\
\hline Dep. ratio $D_{\text {Min }}$ & 0.9025 & 0.9049 & 0.9429 & 0.8701 & 0.8823 & 0.9422 & 0.9582 \\
\hline
\end{tabular}

TABLE 6: Effect of changes in the MTTR of a tyre production system.

\begin{tabular}{lcc}
\hline Subsystem & MTTR at faulty state (hours) & MTTR at ideal state (hours) \\
\hline Banbury mixing & 16.16 & 10.78 \\
Bead and belt cord making & 18.28 & 13.21 \\
Calendaring & 17.55 & 12.38 \\
Bias cutting & 38.46 & 29.34 \\
Extruding & 23.98 & 16.05 \\
Building & 23.59 & 18.09 \\
Curing & 24.38 & 21.15 \\
\hline
\end{tabular}

TABLE 7: Availability variations of the subsystem BC concerning the rate of failure and repair.

\begin{tabular}{lcccccr}
\hline $\begin{array}{l}\lambda_{\mathrm{BC}} \\
\mu_{\mathrm{BC}}\end{array}$ & 0.002 & 0.003 & 0.004 & 0.005 & 0.006 & 0.007 \\
\hline 0.128 & 0.8956 & 0.9015 & 0.9068 & 0.9113 & 0.9154 & 0.9191 \\
0.138 & 0.8653 & 0.8730 & 0.8798 & 0.8858 & 0.8912 & 0.8960 \\
0.148 & 0.8370 & 0.8463 & 0.8544 & 0.8616 & 0.8681 & 0.8740 \\
0.158 & 0.8105 & 0.8211 & 0.8304 & 0.8388 & 0.8463 & 0.8530 \\
0.168 & 0.7857 & 0.7974 & 0.8078 & 0.8171 & 0.8255 & 0.8331 \\
0.178 & 0.7623 & 0.7750 & 0.7863 & 0.7965 & 0.8057 & 0.8140 \\
0.188 & 0.7402 & 0.7538 & 0.7660 & 0.7769 & 0.7868 & 0.8792 \\
MTTR & $\mathbf{7 0 . 1 9 7}$ & $\mathbf{6 5 . 3 2 2}$ & $\mathbf{6 1 . 0 9 6}$ & $\mathbf{5 7 . 4 3 3}$ & $\mathbf{5 4 . 1 9 4}$ & 0.8492 \\
\hline
\end{tabular}

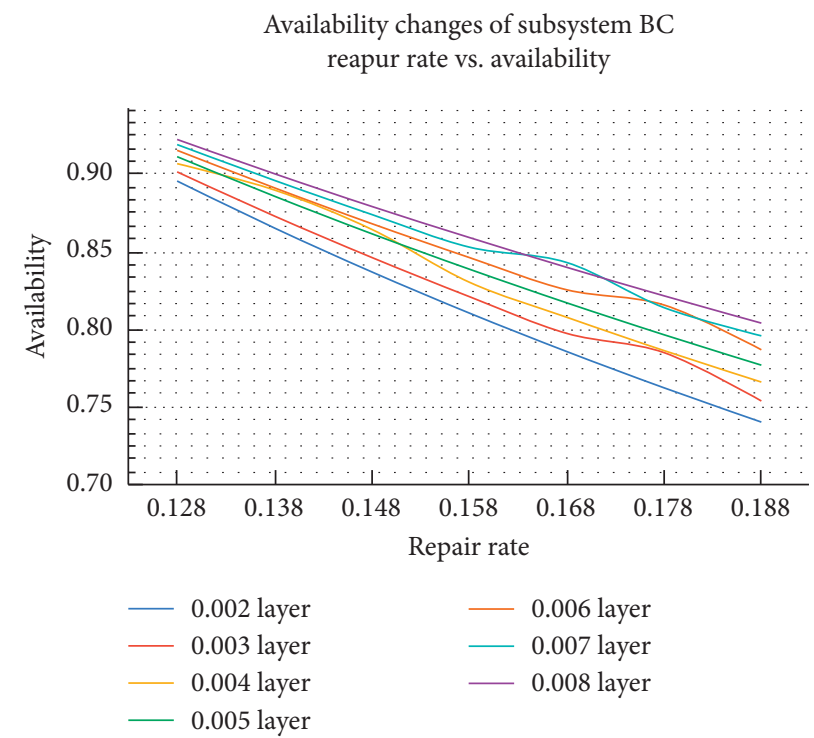

FIgURE 8: Availability variation of the subsystem BC. 
subsystems are having gradual and uniform $A v$ variation while increasing repair and failure rate values of the specific subsystem in the tyre production plant. Figures 5 and 7 reveal the $A v$ and MTTR changes of individual subsystems in the tyre production system of the rubber industry. Table 5 consists of the RAMD indices of the entire subsystem, and based on these results, the subsystem BC has been classified as the most critical subsystem in tyre production. Because of that subsystem $\mathrm{BC}$ having the lowest $A v$, dependability, dependability ratio, and highest MTTR are compared to other subsystems of the tyre production system in the rubber industry.

\section{Conclusion}

In this RAMD analysis of research, the utilization of the MBDA in measuring the $R(t), A v, M(t), d$, and $D_{\min }$ of subsystems to the tyre production plant has been demonstrated. The transition state diagram reveals the relationship between the subsystems in the tyre production plant. From the results of the proposed mathematical modeling and analysis, the most important subsidiary system in the tyre manufacturing system in the rubber industry was identified. The subsystem bias cutting is classified as more sensitive and critical than all other subsystems as it has the lowest $A v$ $0.8387, d 5.19, D_{\text {Min }} 0.8701$, and maximum MTTR of 38.46 hours compared to other subsystems in the tyre manufacturing system. Therefore, it is advisable to monitor the failure and repair rate change of that particular bias cutting subsystem and conclude that the subsystem is very sensitive and highly critical. Then, it is suggested that the maintenance engineer's team of the tyre production plant in the rubber industry should take utmost care and higher attention to maximize the availability, productivity, and effectiveness of the tyre production system of the real-time manufacturing shop floor area of the industry. It is suggested that controlling the maintenance parameter (failure and repair rate) of a more sensitive subsystem through proper maintenance strategy can increase the efficiency of the overall tyre production system in the industry. Finally, the results of this RAMD research are discussed with the maintenance management of the tyre manufacturing plant, as well as to confirm that these proposed structures are highly beneficial to the production and maintenance sectors in the rubber industry.

In the future, this proposed mathematical technology will be used to establish the predictive maintenance management process of smart machines and the health prediction system of its vital components. We intend to implement it with the help of the latest industrial revolution (Industry 4.0) technologies such as Industrial Internet of Things, Cyber-Physical Production Systems, and Internet Communication Technology.

\section{Data Availability}

The detailed input numerical data used in this RAMD analysis of the real-time industrial case study are available from the corresponding author upon request. Due to the actual fact that data were collected from the industry, it is used for research purposes only.

\section{Conflicts of Interest}

The authors declare that there are no conflicts of interest regarding the publication of this research article.

\section{Acknowledgments}

The authors wish to thank the Management of Kalasalingam Academy of Research and Education (KARE), Krishnankovil, Tamil Nadu, India, and Vellore Institute of Technology (VIT), Vellore, Tamil Nadu, India, for their support in this research.

\section{References}

[1] G. Sharma and R. Khanduja, "Performance evaluation and availability analysis of feeding system in a sugar industry," International Journal of Engineering and Applied Sciences, vol. 3, no. 9, pp. 38-50, 2013.

[2] P. Tsarouhas, "Evaluation of reliability, availability and maintainability of a milk production line," International Journal of Industrial and Systems Engineering, vol. 31, no. 3, pp. 324-342, 2019.

[3] P. Tsarouhas, "Statistical techniques of reliability, availability, and maintainability (RAM) analysis in industrial engineering," in Diagnostic Techniques in Industrial Engineering, pp. 207-231, Springer, Berlin, Germany, 2018.

[4] F. Corvaro, G. Giacchetta, B. Marchetti, and M. Recanati, "Reliability, Availability, Maintainability (RAM) study, on reciprocating compressors API 618," Petroleum, vol. 3, no. 2, pp. 266-272, 2017.

[5] S. Mohammad Zadeh Dogahe and S. J. Sadjadi, "A new biobjective model to optimize integrated redundancy allocation and reliability-centered maintenance problems in a system using metaheuristics," Mathematical Problems in Engineering, vol. 2015, Article ID 396864, 16 pages, 2015.

[6] J. P. Vargas, J. C. Koppe, S. Pérez, and J. P. Hurtado, "Planning tunnel construction using Markov chain Monte Carlo (MCMC)," Mathematical Problems in Engineering, vol. 2015, Article ID 797953, 8 pages, 2015.

[7] P. Tsarouhas, "Reliability, availability and maintainability analysis of a bag production industry based on the six sigma DMAIC approach," International Journal of Lean Six Sigma, vol. 12, 2020.

[8] N. Ye, Z.-Q. Wang, R. Malekian, Q. Lin, and R.-C. Wang, “A method for driving route predictions based on hidden Markov model," Mathematical Problems in Engineering, vol. 2015, Article ID 824532, 12 pages, 2015.

[9] X.-Z. Ma and W.-Y. Lv, "Joint optimization of production and maintenance using Monte Carlo method and metaheuristic algorithms," Mathematical Problems in Engineering, vol. 2019, Article ID 3670495, 22 pages, 2019.

[10] M. Ameri, A. Jarrahi, F. Haddadi, and M. H. Mirabimoghaddam, "A two-stage stochastic model for maintenance and rehabilitation planning of pavements," Mathematical Problems in Engineering, vol. 2019, Article ID 3971791, 15 pages, 2019.

[11] A. Jiang, N. Dong, K. L. Tam, and C. Lyu, "Development and optimization of a condition-based maintenance policy with sustainability requirements for production system," 
Mathematical Problems in Engineering, vol. 2018, Article ID 4187575, 19 pages, 2018.

[12] N. Yuguang, S. Wang, and M. Du, "A combined Markov chain model and generalized projection nonnegative matrix factorization approach for fault diagnosis," Mathematical Problems In Engineering, vol. 2017, Article ID 7067025, 7 pages, 2017.

[13] H. Zhao, "High dimension model representation-based Response Surface for reliability analysis of tunnel," Mathematical Problems in Engineering, vol. 2018, Article ID 8049139, 10 pages, 2018.

[14] M. Ruiz, L. Ramirez, F. Navarrina, M. Aymerich, and D. López-Navarrete, "A mathematical model to evaluate the impact of the maintenance strategy on the service life of flexible pavements," Mathematical Problems in Engineering, vol. 2019, Article ID 9480675, 10 pages, 2019.

[15] L. Wang, Q. Yang, and Y. Tian, "Reliability analysis of 6component star Markov repairable system with spatial dependence," Mathematical Problems in Engineering, vol. 2017, Article ID 9728019, 7 pages, 2017.

[16] A. Aggarwal, S. Kumar, and V. Singh, "Performance modeling of the skim milk powder production system of a dairy plant using RAMD analysis," International Journal Of Quality \& Reliability Management, vol. 32, 2015.

[17] A. Kr Aggarwal, S. Kumar, and V. Singh, "Performance modeling of the serial processes in refining system of a sugar plant using RAMD analysis," International Journal Of System Assurance Engineering And Management, vol. 8, pp. 19101922, 2018.

[18] A. K. Agrawal, V. M. S. R. Murthy, and S. Chattopadhyaya, "Investigations into reliability, Maintainability and availability of tunnel boring machine operating in mixed ground condition using Markov chains," Engineering Failure Analysis, vol. 105, pp. 477-489, 2019.

[19] O. Dahiya, A. Kumar, and M. Saini, "Mathematical modeling and performance evaluation of A-pan crystallization system in a sugar industry," SN Applied Sciences, vol. 1, p. 339, 2019.

[20] A. Yaghoubi, S. Rahimi, R. Soltani, and S. T. Akhavan Niaki, "Availability analysis of a cooking oil production line," Journal of Optimization in Industrial Engineering, vol. 14, no. 1, pp. 1-9, 2021.

[21] M. Saini, D. Goyal, A. Kumar, and D. Sinwar, "Investigation of performance measures of power generating unit of sewage treatment plant," Journal of Physics: Conference Series, vol. 1714, no. 1, 2021.

[22] M. Stojanova, T. Begin, and A. Busson, "A Markov model for performance evaluation of channel bonding in IEEE 802.11," Ad Hoc Networks, vol. 115, p. 102449, 2021.

[23] S. Pirbhulal, V. Gkioulos, and S. Katsikas, "A systematic literature review on RAMS analysis for critical infrastructures protection," International Journal of Critical Infrastructure Protection, vol. 33, p. 100427, 2021.

[24] L. Yan, T. Zhang, Y. Gao, R. Wang, and S. Ding, "Reliability analysis of station autonomous computer system based on fuzzy dynamic fault tree and Markov model," Engineering Reports, p. 12376, 2021.

[25] M. Manglik, N. Rawat, and M. Ram, "Reliability and Availability analysis of a cloud computing transition system under multiple failures," International Journal of Quality \& Reliability Management, vol. 37, no. 6/7, pp. 823-835, 2020.

[26] B. Acay, R. Ozarslan, R. Ozarslan, and E. Bas, "Fractional physical models based on falling body problem," AIMS Mathematics, vol. 5, no. 3, pp. 2608-2628, 2020.
[27] B. Acay and M. Inc, "Fractional modeling of temperature dynamics of a building with singular kernels," Chaos, Solitons \& Fractals, vol. 142, p. 110482, 2021.

[28] B. Acay and M. Inc, "Electrical circuits RC, LC, and RLC under generalized type non-local singular fractional operator," Fractal and Fractional, vol. 5, no. 1, p. 9, 2021.

[29] A. Yusuf, B. Acay, U. T. Mustapha, M. Inc, and D. Baleanu, "Mathematical modeling of pine wilt disease with Caputo fractional operator," Chaos, Solitons \& Fractals, vol. 143, p. 110569, 2021.

[30] A. Al-Douri, V. Kazantzi, F. T. Eljack, M. S. Mannan, and M. M. El-Halwagi, "Mitigation of operational failures via an economic framework of reliability, availability, and maintainability (RAM) during conceptual design," Journal of Loss Prevention in the Process Industries, vol. 67, p. 104261, 2020.

[31] P. Pandian, P. Venkumar, and S. Rajakarunakaran, "Fuzzy hybrid decision model for supplier evaluation and selection," International Journal of Production Research, vol. 51, pp. 3903-3919, 2013.

[32] E. R. Barlow and P. Frank, "Mathematical theory of reliability," Society for Industrial and Applied Mathematics, SIAM, Philadelphia, PA, USA, 1996.

[33] K. L. Cavalca, "Availability optimization with genetic algorithm," International Journal of Quality \& Reliability Management, vol. 20, 2003.

[34] R. K. Sharma and S. Kumar, "Performance modeling in critical engineering systems using RAM analysis," Reliability Engineering \& System Safety, vol. 93, no. 6, pp. 913-919, 2008.

[35] J. G. Wohl and G. Joseph, "System operational readiness and equipment dependability," IEEE Transactions on Reliability, vol. R-15, no. 1, pp. 1-6, 1966.

[36] K. Velmurugan, P. Venkumar, and R. Sudhakarapandian, "Design of optimal maintenance policy using Markov model," International Journal of Engineering and Advanced Technology, vol. 9, pp. 907-917, 2019.

[37] K. Velmurugan, P. Venkumar, and R. Sudhakarapandian, "Reliability availability maintainability analysis in forming industry," International Journal of Engineering and Advanced Technology, vol. 9, pp. 822-828, 2019. 